CRORR 9 (2018), 293-299

\title{
Kernel-like Search for Robust Emergency System Designing
}

\author{
Marek Kvet ${ }^{1, *}$ and Jaroslav Janáček ${ }^{1}$ \\ ${ }^{1}$ Faculty of Management Science and Informatics, University of Žilina \\ Univerzitná 8215/1, 01026 Žilina, Slovakia \\ E-mail: $\{\{$ marek.kvet, jaroslav.janacek\}@fri.uniza.sk\}
}

\begin{abstract}
Emergency service system, which satisfies randomly emerging demands of public for necessary treatment, is determined by deployment of limited number of service centers at positions from a given set of possible locations. The objective is to minimize average response time of the nearest ambulance vehicle usually located at a service center. The robust service system is designed to comply with specified scenarios by minimizing the maximal value of the above mentioned objective functions corresponding to the particular scenarios, which represent consequences of random failures in the road network. The detrimental events may correspond to congestion, disruptions or blockages of roads. The robust emergency system design problem can be modelled by means of mathematical programming. The model includes scenarios and the associated link-up constraints, which connect average response time connected with individual scenarios to the general objective function, which is maximum of these objective functions. The min-max link-up constraints and the cardinality of the scenario set represent an undesirable burden in any solving process used for design solution. Within this paper, we present a kernel-like search algorithm, which tries to replace the solving process of the huge problem above by a series of smaller problems, which deal with either small subset of scenarios or reduced set of possible center locations.
\end{abstract}

Keywords: kernel-like search, robust emergency system design, detrimental scenarios

Received: July 23, 2018; accepted: November 14, 2018; available online: December 13, 2018

DOI: 10.17535 /crorr.2018.0023

\section{Introduction}

This paper deals with the emergency service system designing to satisfy the future demands of the system users in case of emergency. As the modelled decisions of the optimal service center deployment represent a strategic decision-making problem, several factors influencing the resulting system design should be taken into account. None of those factors consists in system robustness [1], [10], [11], and [12].

A robust design of an emergency service system operating on a real transportation network has to be resistant to randomly appearing failures in the network. To incorporate system resistance into the associated mathematical model, a finite set of failure scenarios is generated to cover the most fatal combinations of the failures. Generating the scenario set is a complex problem, which plays an important role in the robust system designing. A suitable detrimental scenario set can be formed either by experts in the transportation science based on their experience or it can result from the road network analysis as suggested in [7]. Then, the system is suggested in such a way that the maximal detrimental impact of the individual scenarios is minimized, i.e. the maximal value of objective functions corresponding with the individual scenarios is minimized. A simple emergency system design can be modelled as a weighted $p$ -

\footnotetext{
* Corresponding author.
} 
median problem, because the sources, from which the associated service can be provided, are limited [2], [4]. While a simple weighted $p$-median problem is easily solvable, the robust system design is hard to be solved due to the resulting model size and usage of min-max constraints, which link up the individual scenario objective functions to their common upper bound, which represents the objective function of the robust design problem. Therefore, many different modelling and solving techniques to the robust service system designing have been recently developed [5], [9], [10].

Presented research was evoked by the Kernel-Search approach, which proved to be a sufficient tool for approximate solving of large mathematical programming problems [3], [8], and [13]. The Kernel-Search approach is based on replacing the solving process of a large problem by a series of smaller sub-problems. The original problem decomposition is based on solving LP-relaxation of the original model and importance accessing of the individual variables. The presented kernel-like approach avoids the LP-relaxation and uses other way of variable importance evaluation to obtain a good robust design of the emergency system.

The remainder of this paper is organized as follows: Section 2 contains the description of the exact approach to robust emergency service system design problem. This approach can be accelerated by fixing some decision variables either to one or to zero. In Section 3, suggested approximate approach is explained. The fourth Section contains an overview of performed numerical experiments and yields a brief comparative analysis of center deployments. Finally, the obtained findings and future research directions are summarized in Section 5.

\section{Robust emergency system design problem}

A model of the robust emergency system design problem can be built using the following input data and decision variables. Symbols $J$ and $I$ are used to denote the sets of users' locations and possible service center locations respectively. In the set $I$, we will distinguish three subsets $F 1, F 0$ and $V$. Set $F 1$ contains the locations, in which a service center must be located. Set $F 0$ consists of center locations, where no center is allowed to be located, and $V$ is the set of possible locations, from which $p$ service centers must be chosen. Symbol $b_{j}$ stands for the number of users sharing the location $j$. The set of considered failure scenarios is denoted by symbol $U$. The response time of the service center $i$ for a user $j$ under a specific scenario $u \in U$ follows from the distance between locations $i$ and $j$ and is denoted as $d_{i j u}$. In this paper, we assume only integer values of $d_{i j u}$. Furthermore, let us introduce the symbol $v+1$ to denote the maximal response time from all matrices $\left\{d_{i j u}\right\}$ corresponding to detrimental scenarios $u \in U$, i.e. $v+1=\max \left\{d_{i j u}: i \in I, j \in J, u \in U\right\}$. Knowledge of the value $v$ is necessary for the radial mathematical model formulation.

The objective of the robust system design problem is to minimize the upper bound of all objective functions of the individual scenarios. In this paper, we present the radial formulation of the problem, which could considerably accelerate the associated solving process [9].

Further, we introduce the following decision variables. The variable $y_{i} \in\{0,1\}$ models the decision on service center locating at each location $i \in V$. Variable $y_{i}$ takes the value of 1 if a service center is located at $i$ and it takes the value of 0 otherwise. In problem formulation below, the variable $h$ denotes the upper bound of the objective functions across the scenario set $U$. Auxiliary binary variables $x_{j s u}$ for each $s=0 \ldots v$ and $u \in U$ were introduced to complete the radial model. Variable $x_{j s u}$ takes the value of 1 , if the response time of the nearest service center to the user at $j \in J$ under the scenario $u \in U$ is greater than $s$ and it takes the value of 0 otherwise. Next, we introduce a zero-one constant $a_{i j u}{ }^{s}$ for each quadruple $(u, i, j, s)$, where $u \in U$, $i \in U F 1, j \in J, s \in[0 . . v]$. The constant $a_{i j u}{ }^{s}$ is equal to 1 , if the response time $d_{i j u}$ of a center located at $i$ on a user located at $j$ is less than or equal to $s$, otherwise $a_{i j u}{ }^{s}$ is equal to 0 . Then the radial model of the problem follows. 


$$
\begin{gathered}
\text { Minimize } \\
\text { Subject to: } \quad x_{j s u}+\sum_{i \in V} a_{i j u}^{s} y_{i}+\sum_{i \in F 1} a_{i j u}^{s} \geq 1 \quad \text { for } j \in J, s=0,1, \ldots, v, u \in U \\
\sum_{i \in V} y_{i}=p \\
\sum_{j \in J} b_{j} \sum_{s=0}^{v} x_{j s u} \leq h \quad \text { for } u \in U \\
y_{i} \in\{0,1\} \quad \text { for } i \in V \\
x_{j s u} \in\{0,1\} \quad \text { for } j \in J, s=0,1, \ldots, v, u \in U
\end{gathered}
$$

The objective function $h$ in (1) represents the upper limit of all objective function values across the individual scenarios. The constraints (2) assure that the variable $x_{j s u}$ is allowed to take the value of 0 only if at least one center is located in radius $s$ from the user location $j$. The constraint (3) forces exactly $p$ service centers to be located in the set of locations $V$. The link-up constraints (4) ensure that each individual scenario objective function is less than or equal to the limiting variable $h$. For purpose of conciseness, we introduce the denotation of the set of resulting (optimal) service center locations as $\operatorname{IR}(U, F 1, F 0, p)$. The set includes service centers from both $F 1$ and $V$.

\section{Approximate approach to the robust service system design}

The suggested kernel-like search algorithm is based on step-by-step adjustment of sets of fixed decision variables. For the formulation of the suggested approximate algorithm, we employ the procedure $I R(U, F 1$, $F 0, p)$ introduced in previous section. We also use the data structures $J,\left\{b_{j}\right\}, I, U,\left\{d_{i j u}\right\}$ introduced there and assume that the number $p$ of centers to be additionally located in the set $V=I-F 1-F 0$ is known. We note that the procedure $\operatorname{IR}(\{u\}, F 1, F 0, p)$ performed with set of scenarios $\{u\}$ containing only one scenario $u$ gives the optimal classical emergency service system design for the given scenario. It is assumed that the set $U$ of scenarios contains one special scenario $b$ corresponding to standard conditions and the other scenarios, which correspond to detrimental events.

Inputs of the following algorithm are represented by the scenario set $U$, set $F 1$ containing the locations, in which a service center must be located and set $F 0$ containing the center locations, where no center is allowed to be located. The algorithm operations are controlled using two integer threshold parameters $t 1$ and $t 0$ from the range $1 \ldots|U|$.

1. Perform the procedure $\operatorname{IR}(\{u\}, F 1, F 0, p)$ for each $u \in U$ and compute the objective function value for each obtained deployment. Order the set of scenarios into sequence $u_{1}, u_{2} \ldots u_{U} \mid$ so that the first scenario is the basic scenario $b$ and the detrimental scenarios follow according to their decreasing objective function value.

2. Initialize the auxiliary structures $r=p, C 1=F 1, C 0=F 0$, Score $1(i)=0$ and $S \operatorname{core} O(i)=0$ for each $i \in I-F 1$ Fo.

3. For $k=1 \ldots|U|$ repeat step 3 and then terminate. The resulting deployment is contained in the set $L$.

4. Compute $L=I R\left(\left\{u_{1}, u_{2}, \ldots, u_{k}\right\}, C 1, C 0, r\right)$, update scores for each $i \in I$-C1-C0 according to the rule: If $i \in L$ then $\operatorname{Score} 1(i)=S \operatorname{core} 1(i)+1$, else $\operatorname{Score} O(i)=S \operatorname{Score} O(i)+1$. Update $C 0$ and $C 1$ according to the 
following rule applied on each $i \in I$-C1-C0. If Score1 $(i)$ - ScoreO $(i)>t 1$, then $C 1=C 1 \cup\{i\}$ else if ScoreO $(i)-\operatorname{Score} 1(i)>t 0$, then $C 0=C \backsim\{i\}$. Update $r=|L|-|C 1|$.

The objective function value of the resulting center deployment $L$ can be enumerated according to (8).

$$
f(L)=\max \left\{\sum_{j \in J} b_{j} * \min \left\{d_{i j u}: i \in L\right\}: u \in U\right\}
$$

\section{Computational study and results}

Computational study reported in this section was aimed at ascertainment of the characteristics of the suggested approach from the viewpoint of computational time and the resulting system design. To solve the problems described in previous sections, the optimization software FICO Xpress 8.3 (64-bit, release 2017) was used and the experiments were run on a $\mathrm{PC}$ equipped with the Intel® Core ${ }^{\mathrm{TM}}$ i7 $5500 \mathrm{U}$ processor with the parameters: $2.4 \mathrm{GHz}$ and $16 \mathrm{~GB}$ RAM. Benchmarks used in this study were derived from the real emergency service system originally implemented in selected regions of Slovak Republic. For selected selfgoverning regions, i.e. Bratislava (BA), Banská Bystrica (BB), Košice (KE), Nitra (NR), Prešov (PO), Trenčín (TN), Trnava (TT) and Žilina (ZA), all cities and villages with corresponding number $b_{j}$ of inhabitants were taken into account. The coefficients $b_{j}$ were rounded to hundreds. These sub-systems cover demands of all communities - towns and villages spread over the particular regions by given number of ambulance vehicles. Here, the set of communities represents both the set $J$ of users' locations and the set $I$ of possible center locations. For each problem instance, ten detrimental scenarios were generated according to the random matrix row selection described in [5] and [6].

\begin{tabular}{|c|c|c|c|c|}
\hline Region & $|I|$ & $p$ & $C T$ & ObjFrobust \\
\hline BA & 87 & 9 & 52.8 & 25417 \\
\hline BB & 515 & 52 & 1605.0 & 18549 \\
\hline KE & 460 & 46 & 1235.5 & 21286 \\
\hline NR & 350 & 35 & 11055.1 & 24193 \\
\hline PO & 664 & 67 & 3078.2 & 21298 \\
\hline TN & 276 & 28 & 616.6 & 17535 \\
\hline TT & 249 & 25 & 563.8 & 20558 \\
\hline ZA & 315 & 32 & 1304.7 & 23004 \\
\hline
\end{tabular}

Table 1: Results of the exact approach for robust service system designing applied on the selfgoverning regions of Slovakia

An individual experiment was organized so that the optimal robust service system design using the exact approach described by the model (1) - (7) was obtained first. The sets $F 1$ and $F 0$ were empty. The optimal solution of each solved instance is reported in Table 1, which contains also the cardinality of possible service center location set $I$ and the number $p$ of centers to be located. The computational time in seconds is reported in the column denoted by $C T$ and the optimal objective function values (1) are given in the last column of the table denoted by ObjFrobust. 


\begin{tabular}{|c|c|c|c|c|c|c|}
\hline Region & $t 1$ & to & $C T$ & ObjF $F^{\text {approx }}$ & gap [\%] & $H D$ \\
\hline \multirow[t]{5}{*}{$\mathrm{BA}$} & 2 & 2 & 3.9 & 26980 & 6.15 & 4 \\
\hline & 3 & 3 & 11.0 & 28963 & 13.95 & 6 \\
\hline & 4 & 4 & 19.4 & 28963 & 13.95 & 6 \\
\hline & 5 & 5 & 36.1 & 28963 & 13.95 & 6 \\
\hline & 6 & 6 & 53.0 & 26023 & 2.38 & 6 \\
\hline \multirow[t]{5}{*}{ BB } & 2 & 2 & 94.0 & 19159 & 3.29 & 18 \\
\hline & 3 & 3 & 328.5 & 18939 & 2.10 & 10 \\
\hline & 4 & 4 & 454.9 & 18783 & 1.26 & 8 \\
\hline & 5 & 5 & 657.6 & 18741 & 1.04 & 12 \\
\hline & 6 & 6 & 926.8 & 18741 & 1.04 & 12 \\
\hline \multirow[t]{5}{*}{$\mathrm{KE}$} & 2 & 2 & 90.7 & 24478 & 15.00 & 20 \\
\hline & 3 & 3 & 278.4 & 24170 & 13.55 & 16 \\
\hline & 4 & 4 & 436.8 & 24170 & 13.55 & 16 \\
\hline & 5 & 5 & 847.6 & 24225 & 13.81 & 16 \\
\hline & 6 & 6 & 1456.6 & 24213 & 13.75 & 14 \\
\hline \multirow[t]{5}{*}{$\mathrm{NR}$} & 2 & 2 & 46.5 & 24297 & 0.43 & 10 \\
\hline & 3 & 3 & 135.4 & 24273 & 0.33 & 10 \\
\hline & 4 & 4 & 313.1 & 24335 & 0.59 & 6 \\
\hline & 5 & 5 & 628.5 & 24203 & 0.04 & 8 \\
\hline & 6 & 6 & 1913.5 & 24203 & 0.04 & 8 \\
\hline \multirow[t]{5}{*}{$\mathrm{PO}$} & 2 & 2 & 227.0 & 21598 & 1.41 & 12 \\
\hline & 3 & 3 & 618.0 & 21390 & 0.43 & 10 \\
\hline & 4 & 4 & 1019.2 & 21298 & 0.00 & 0 \\
\hline & 5 & 5 & 1543.5 & 21298 & 0.00 & 0 \\
\hline & 6 & 6 & 2758.7 & 21298 & 0.00 & 0 \\
\hline \multirow[t]{5}{*}{$\mathrm{TN}$} & 2 & 2 & 35.0 & 18186 & 3.71 & 12 \\
\hline & 3 & 3 & 81.5 & 17907 & 2.12 & 8 \\
\hline & 4 & 4 & 250.8 & 17648 & 0.64 & 12 \\
\hline & 5 & 5 & 364.0 & 17558 & 0.13 & 10 \\
\hline & 6 & 6 & 535.4 & 17558 & 0.13 & 10 \\
\hline \multirow[t]{5}{*}{$\mathrm{TT}$} & 2 & 2 & 21.1 & 21812 & 6.10 & 12 \\
\hline & 3 & 3 & 94.9 & 21062 & 2.45 & 6 \\
\hline & 4 & 4 & 174.1 & 21043 & 2.36 & 8 \\
\hline & 5 & 5 & 247.7 & 20597 & 0.19 & 6 \\
\hline & 6 & 6 & 566.4 & 20558 & 0.00 & 0 \\
\hline \multirow[t]{5}{*}{$\mathrm{ZA}$} & 2 & 2 & 68.6 & 23787 & 3.40 & 14 \\
\hline & 3 & 3 & 101.6 & 23448 & 1.93 & 8 \\
\hline & 4 & 4 & 133.2 & 23159 & 0.67 & 6 \\
\hline & 5 & 5 & 180.2 & 23156 & 0.66 & 8 \\
\hline & 6 & 6 & 299.8 & 23080 & 0.33 & 10 \\
\hline
\end{tabular}

Table 2: Results of the approximate kernel-like approach to robust service system design with different values of parameters t1 and to applied on the self-governing regions of Slovakia

After obtaining the optimal solution of the exact approach, the approximate algorithm was applied. For each self-governing region, five settings of parameters $t 1$ and $t 0$ were studied. These values are reported in the left part of Table 2. The right part of the table contains the studied characteristics of suggested 
approximate algorithm. The computational time in seconds is reported in the column denoted by $C T$. The next column denoted by $O b j F^{a p p r o x}$ contains the objective function value resulting from the approximate approach. For better comparison, the value of gap from the exact solution was computed. Generally, gap is defined as the difference between two objective function values. Here, the result of the exact approach was taken as the base and thus, gap is reported in percentage of this value. Finally, the resulting system designs are compared by Hamming distance $H D$ of the associated vectors of location variables $y_{i}$. Hamming distance of two vectors $\mathbf{y}^{\mathrm{a}}$ and $\mathbf{y}^{\mathrm{b}}$ is defined by (9).

$$
H D\left(\mathbf{y}^{\mathbf{a}}, \mathbf{y}^{\mathbf{b}}\right)=\sum_{i \in I}\left(y_{i}^{\mathbf{a}}-y_{i}^{\mathbf{b}}\right)^{2}
$$

In the tables reporting performed numerical experiments we can observe that presented approach enables to get the resulting robust service system design much faster than the original exact approach. As far as the solution accuracy is concerned, it must be noted that the approximate algorithm is sensitive to the parameters settings. The higher the parameters are, the bigger model is solved in each step of the algorithm and the variables are fixed to their values much later. Obviously, higher values of mentioned parameters mean also better solution accuracy, but it is paid by higher computational time. Therefore, we suggest setting the parameters at the value 4 or 5 . Thus, we can conclude that the suggested approach provides us with very good results with exception of the self-governing region Košice (KE). This irregularity will be analyzed and studied in the future research.

\section{Conclusions}

The main research goal of this paper was aimed at effective emergency service system designing. The necessity of making the system resistant to randomly occurring detrimental events has led to the concept of robustness and introducing the set of scenarios. Since the associated mathematical model takes the form of a min-max problem, which is hard to be solved, many different approximate approaches have been developed. In this paper, we have introduced a kernel-like search algorithm, which tries to replace the original solving process by a series of easier problems, which deal with either smaller subset of scenarios or a reduced set of possible center locations. Based on reported results we can conclude that we have presented a promising way of robust service system designing.

Future research in this field could be aimed at development of such new approximate solving techniques, which would reach a near-optimal solution in a short time. Another research goal could be focused on mastering the presented problem with larger set of detrimental scenarios.

\section{Acknowledgement}

This work was supported by the research grants VEGA 1/0342/18 "Optimal dimensioning of service systems", VEGA 1/0463/16 "Economically efficient charging infrastructure deployment for electric vehicles in smart cities and communities" and APVV-15-0179 "Reliability of emergency systems on infrastructure with uncertain functionality of critical elements".

\section{References}

[1] Correia, I. and Saldanha da Gama, F. (2015). Facility locations under uncertainty. In Laporte, G. Nikel, S. and Saldanha da Gama, F. (Eds). Location Science (pp. 177-203). Heidelberg: Springer Verlag. 
[2] Current, J., Daskin, M. and Schilling, D. (2002). Discrete network location models. In Drezner Z. et al. (Eds). Facility location. Applications and theory (pp 81-118). Berlin: Springer Verlag.

[3] Guastaroba, G., Savelsbergh, M. and Speranza, M. G. (2017). Adaptive Kernel Search: A heuristic for solving Mixed Integer linear Programs. European Journal of Operational Research, 263(3), pp. 789804.

[4] Ingolfsson, A., Budge, S. and Erkut, E. (2008). Optimal ambulance location with random delays and travel times. Health Care Management Science, 11(3), 262-274.

[5] Janáček, J. and Kvet, M. (2017). An Approach to Uncertainty via Scenarios and Fuzzy Values. Croatian Operational Research Review 8 (1), 237-248.

[6] Janáček, J. and Kvet, M. (2017). Effectiveness of the hill-climbing algorithm for robust emergency system design. In Proceedings of the 12th international conference Strategic management and its support by information systems, May $25^{\text {th }}-26^{\text {th }} 2017$, Ostrava, Czech Republic, (pp. 247-254).

[7] Janáček, J. and Kvet, M. (2018). Detrimental Scenario Construction Based on Network Link Characteristics. In Proceedings of the 19th International Carpathian Control Conference (ICCC) 2018, Szilvasvarad, Hungary, (pp. 629-632).

[8] Jánošíková, L. (2018). Kernel Search for the Capacitated p-Median Problem. In Proceedings of the International Scientific Conference QUANTITATIVE METHODS IN ECONOMICS: Multiple Criteria Decision Making XI, May $23^{\text {rd }}-25^{\text {th }} 2018$, Trenčianske Teplice, Slovakia, (pp. 158-164).

[9] Kvet, M. and Janáček, J. (2017). Hill-Climbing Algorithm for Robust Emergency System Design with Return Preventing Constraints. In $9^{\text {th }}$ International Conference on Applied Economics: Contemporary Issues in Economy, 2017, Toruń, Poland, pp. 156-165.

[10] Kvet, M. and Janáček, J. (2017). Struggle with curse of dimensionality in robust emergency system design. In Proceedings of the $35^{\text {th }}$ international conference Mathematical Methods in Economics MME 2017, September $13^{\text {th }}-15^{\text {th }} 2017$, Hradec Králové, Czech Republic, (pp. 396-401).

[11] Pan, Y., Du, Y. and Wei, Z. (2014). Reliable facility system design subject to edge failures. American Journal of Operations Research 4, 164-172.

[12] Scaparra, M.P., Church, R.L. (2015). Location Problems under Disaster Events. Location Science, eds. Laporte, Nikel, Saldanha da Gama, 623-642.

[13] Stefanello, F., Araújo, O. and Müller, F. (2015). Matheuristics for the capacitated p-median problem. International Transactions in Operational Research, 22(1), pp. 149-167. 